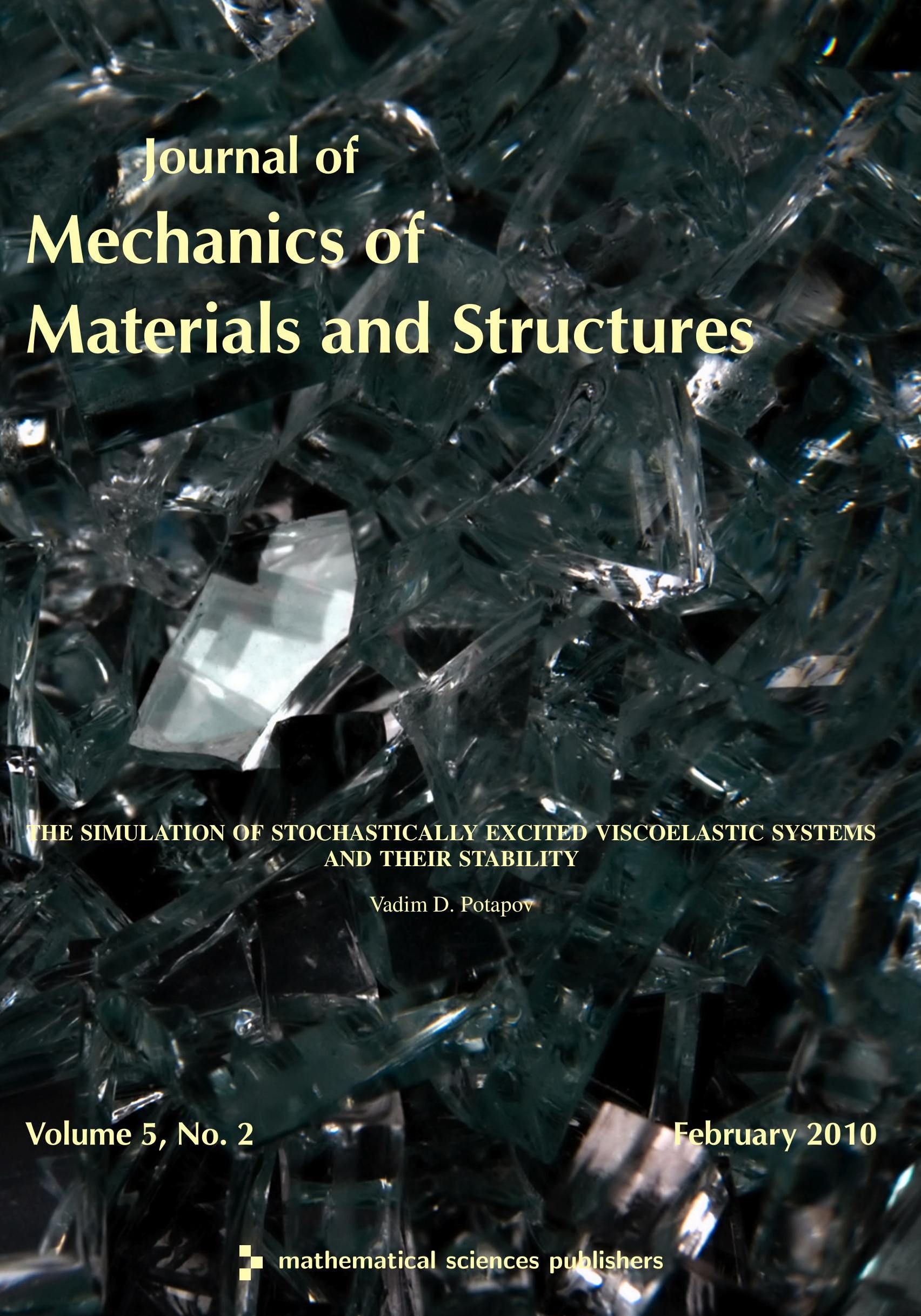




\title{
THE SIMULATION OF STOCHASTICALLY EXCITED VISCOELASTIC SYSTEMS AND THEIR STABILITY
}

\author{
VADIM D. POTAPOV
}

\begin{abstract}
An efficient method for the analysis of nonlinear elastic and viscoelastic systems under the action of parametric forces in the form of Gaussian random stationary processes is suggested. The spectral densities of the input random stationary processes are assumed to be in the form of rational functions. The method is based on the simulation of stochastic processes and the numerical solution of differential equations, describing the motion of the system. Considering a sample of solutions, statistical characteristics of trajectories can be found. The effect of the parameters of the input random processes on the indicated statistical characteristics is investigated. Special attention is devoted to investigation of the stability of the unperturbed motion of elastic and viscoelastic systems. To analyze the stability of the unperturbed motion of the system the motion due to perturbations of the initial conditions is considered. The method of the stability investigation is based on the numerical solution of differential equations, describing the perturbed motion of the system, and the calculation of the top Lyapunov exponents. The method results in the estimation of the stability with respect to statistical moments of different orders. In some cases the superposition of a stochastic noise on the deterministic periodic excitation can have a stabilizing effect on the motion of elastic and viscoelastic systems.
\end{abstract}

\section{Introduction}

The behavior of nonlinear mechanical systems subjected to random loads in the form of random stationary processes, was considered in [Dimentberg 1980; 1988; Simiu 2002], which contain an extensive review of investigations in the indicated direction. As a rule, these investigations were developed under various restrictions imposed on the character of the stationary processes. For example, sometimes these processes were assumed as narrow-band; in this case the method used for the solution was similar to the method of the harmonic balance. In other cases the level of the nonlinearity was assumed to be small, and then the method of stochastic averaging was applied [Dimentberg 1980; 1988]. For the estimation of the reliability of nonlinear systems in [Simiu 2002] the Melnikov stochastic process was used.

Some questions on the stability of elastic and viscoelastic systems, subjected to random loads in the form of random stationary processes, were considered in [Potapov 1999]. A numerical method was suggested for nonlinear problems using the method of canonical expansions of stationary processes. The present paper is devoted to a numerical analysis of nonlinear oscillation of viscoelastic systems under stochastic excitation in the form of a Gaussian stationary process with a rational spectral density. The analysis is based on numerical simulation of the input stationary process, on a numerical solution of

Keywords: stochastics, viscoelasticity, nonlinear oscillations, stability, simulation, top Lyapunov exponents, random stationary processes.

Financial support by the Russian Foundation of Basic Researches under grant N 09-01-00267 is gratefully acknowledged by the author. 
the differential equations which describe the motion of the system, and, in the case of the stability investigation of this motion, on the calculation of the top Lyapunov exponent. In the example of a plate subjected to a random stationary load acting in the middle plane, peculiarities in the application of the proposed method are considered. Particular attention is devoted to the interaction of deterministic periodic and stochastic excitations from the stability point of view of the motion. It is shown that in some cases the superposition of a colored noise can have a stabilizing effect on an unstable deterministic system.

\section{Statement of the problem}

The dynamic behavior of a viscoelastic system with regard to finite deflections, provided that strains are small, is described by a system of nonlinear integrodifferential equations with partial derivatives. Using different methods, for example, the method of finite elements, the method of finite differences, the Bubnov-Galerkin method, et cetera, this system can be replaced by a system of ordinary integrodifferential equations. The relaxation kernels of the material are assumed as degenerate. This means that they can be presented in the form

$$
R(t, \tau)=\sum_{i=1}^{m} f_{i}(t) \varphi_{i}(\tau)
$$

We increase the dimensions of the phase space and replace the system of integrodifferential equations with a system of nonlinear first-order differential equations:

$$
\dot{\boldsymbol{z}}=\boldsymbol{F}(\boldsymbol{z}, \alpha(t), t),
$$

where $z$ is the vector of unknowns, $\alpha(t)=\alpha^{*}(t)+\alpha^{o}(t), \alpha^{*}(t)$ is a deterministic function, $\alpha^{o}(t)$ is a random stationary process, and $t$ is the time. A dot indicates the derivative with respect to time $t$.

The random stationary process $\alpha^{o}(t)$ is assumed to be a Markov process, which is a result of passing the Gaussian white noise through a linear filter of the $m$-th order. That is, the function $\alpha^{o}(t)$ is the solution of the stochastic differential equation

$$
\alpha^{o^{(m)}}+d_{1} \alpha^{\alpha^{(m-1)}}+\cdots+d_{m-1} \dot{\alpha}^{o}+d_{m} \alpha^{o}=h \xi(t),
$$

where $d_{k}(k=1,2, \ldots, m)$ and $h$ are constants, and $\xi(t)$ is the Gaussian white noise.

For the analysis of the behavior of the system the method of statistical simulation is used, which is based on the numerical solution of differential equations (by the Runge-Kutta method) in combination with a numerical method of obtaining the realizations of random stationary processes.

\section{Oscillations of the plate under the action of a random load in the middle of the plane}

As an illustration of the present method let us consider transverse oscillations of a thin rectangular viscoelastic plate hinged along all edges and subjected to a uniformly distributed load applied to two opposite edges in the plate plane (see Figure 1). It is assumed that the opposite edges remain parallel to each other during the motion of the plate.

If the material of the plate is isotropic and the Poisson coefficient is constant with respect to time $\mu$, then the equations of the plate oscillations for the case of finite deflections of von Karman type is written 


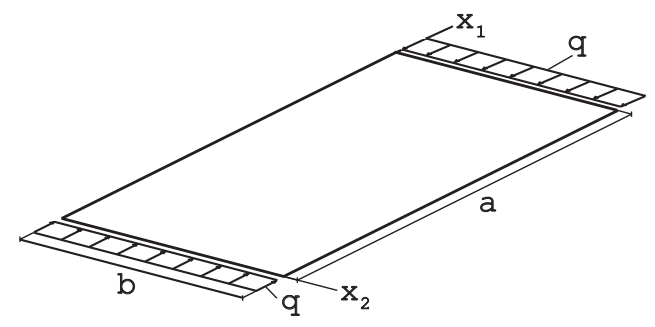

Figure 1. The rectangular plate under loads applied to the middle of the plane.

in the following form [Vol'mir 1967]:

$$
\begin{gathered}
D(1-\boldsymbol{R}) \nabla^{4} w-h\left(\Phi_{, 22} w_{, 11}-2 \Phi_{, 12} w_{, 12}+\Phi_{, 11} w_{, 22}\right)=-\gamma \ddot{w}-k \dot{w}, \\
\frac{1}{E} \nabla^{4} \Phi=(\boldsymbol{I}-\boldsymbol{R})\left(w_{, 12}^{2}-w_{, 11} w_{, 22}\right),
\end{gathered}
$$

where $w$ is the deflection of the plate, $\Phi$ is the function of stresses, acting in the middle of the surface of the plate, $h$ is the thickness of the plate, $\gamma$ is the mass per unit area of the plate, $k$ is the damping coefficient, $\nabla^{4}$ is the biharmonic operator, $E$ is the Young modulus, and $\boldsymbol{R}$ is the relaxation operator.

If the form of the plate is close to square and the initial conditions have the form

$$
\left.w\left(t, x_{1}, x_{2}\right)\right|_{t=0}=f_{0} \sin \frac{\pi}{a} x_{1} \sin \frac{\pi}{b} x_{2},\left.\quad \dot{w}\left(t, x_{1}, x_{2}\right)\right|_{t=0}=v_{0} \sin \frac{\pi}{a} x_{1} \sin \frac{\pi}{b} x_{2},
$$

then the deflection of the plate can be found in a similar form:

$$
w\left(t, x_{1}, x_{2}\right)=f(t) \sin \frac{\pi}{a} x_{1} \sin \frac{\pi}{b} x_{2} .
$$

Really, even for the initial conditions given by (5), the solution of (3) and (4) has a more complicated form. However, since we focus on the qualitative aspect of the problem rather than the quantitative one, we restrict the consideration to the first approximation of the function $w\left(t, x_{1}, x_{2}\right)$, given by (6).

Substituting (6) in the right-hand side of (4) and solving it with respect to the function $\Phi$, we obtain

$$
\Phi\left(t, x_{1}, x_{2}\right)=\frac{1}{32} E(1-\boldsymbol{R}) f^{2}(t)\left(\frac{a^{2}}{b^{2}} \cos \frac{2 \pi}{a} x_{1}+\frac{b^{2}}{a^{2}} \cos \frac{2 \pi}{b} x_{2}\right)-\frac{q x_{1}^{2}}{2 h} .
$$

It is not difficult to prove that the boundary conditions concerning the parallelism of opposite edges are fulfilled [Potapov 1999].

To find the plate deflection amplitude $f(t)$ let us use the Bubnov-Galerkin method. To this end we multiply both sides of (3) by $\sin (\pi / a) x_{1} \sin (\pi / b) x_{2}$ and integrate the resultant relation over the plate area. Then we obtain the integrodifferential equation

$$
z^{\prime \prime}+2 \varepsilon z^{\prime}+(1-\boldsymbol{R}) z-\alpha z+\frac{3}{4}\left(1-\mu^{2}\right) \frac{a^{4}+b^{4}}{\left(a^{2}+b^{2}\right)^{2}} z(1-\boldsymbol{R}) z^{2}=0 .
$$

Here $z=f / h, 2 \varepsilon=k /(\gamma \omega)$, and $\omega$ is the fundamental frequency of plate oscillations, given by

$$
\omega^{2}=\frac{D}{\gamma}\left(\frac{\pi^{2}}{a^{2}}+\frac{\pi^{2}}{b^{2}}\right)^{2}, \quad \alpha=\frac{\pi^{2} q}{D b^{2}}\left(\frac{\pi^{2}}{a^{2}}+\frac{\pi^{2}}{b^{2}}\right)^{-2},
$$


where the prime denotes the derivative with respect to dimensionless time $t_{1}=\omega t$.

If $a=b$ and $\mu=0.3$, then Equation (8) acquires the form

$$
z^{\prime \prime}+2 \varepsilon z^{\prime}+(1-\boldsymbol{R}) z-\alpha z+0.34125 z(1-\boldsymbol{R}) z^{2}=0 .
$$

Suppose that the material of the plate is standard. Then the relaxation kernel $R(t-\tau)$ has an exponential form, $R(t-\tau)=\chi L e^{-\chi(t-\tau)}$, where $\chi$ and $L$ are constant.

We introduce the new variables

$$
z_{1}=z, \quad z_{2}=z^{\prime}, \quad z_{3}=\int_{0}^{t} \chi L e^{-\chi(t-\tau)} z(\tau) d \tau, \quad z_{4}=\int_{0}^{t} \chi L e^{-\chi(t-\tau)} z^{2}(\tau) d \tau,
$$

and we replace the integrodifferential equation (9) by the system of first-order differential equations

$$
\begin{array}{ll}
z_{1}^{\prime}=z_{2}, & z_{2}^{\prime}=-2 \varepsilon z_{2}-(1-\alpha) z_{1}+z_{3}-0.34125 z_{1}\left(z_{1}^{2}-z_{4}\right), \\
z_{3}^{\prime}=\chi L z_{1}-\chi z_{3}, & z_{4}^{\prime}=\chi L z_{1}^{2}-\chi z_{4} .
\end{array}
$$

The solution of these equations should satisfy the initial conditions

$$
z_{1}(0)=\frac{f_{0}}{h}, \quad z_{2}(0)=\frac{v_{0}}{h}, \quad z_{3}(0)=z_{4}(0)=0 .
$$

Let us express the function $\alpha(t)$ in the form of the sum $\alpha(t)=\alpha_{0}+\alpha_{1} \sin \omega t+\alpha^{o}(t)$, where $\alpha_{0}$ and $\alpha_{1}$ are deterministic constants, $\omega$ is the frequency of the deterministic periodic part of the load, $\alpha^{o}(t)$ is a stationary random process with zero mathematical expectation, $\left\langle\alpha^{o}(t)\right\rangle=0$, and the correlation function

$$
K\left(t_{1}-t_{2}\right)=\sigma^{2} \exp \left(-\delta\left|t_{1}-t_{2}\right|\right)\left[\cos \theta\left(t_{1}-t_{2}\right)+\frac{\delta}{\theta} \sin \theta\left(t_{1}-t_{2}\right)\right],
$$

where $\sigma^{2}$ is the dispersion of the process and $\delta$ and $\theta$ are parameters, characterizing the scale of the correlation and the frequency of the implicit periodicity respectively. Angle brackets denote the operation of the mathematical expectation.

The spectral density $S(\omega)$ in this case has the form

$$
S(\omega)=\frac{2 \sigma^{2} \delta}{\pi} \frac{\delta^{2}+\theta^{2}}{\left(\omega^{2}-\theta^{2}-\delta^{2}\right)^{2}+4 \delta^{2} \omega^{2}} .
$$

The considered random process is differentiable.

Equation (2) is written in the following way [Vol'mir 1967; Shalygin and Palagin 1986]:

$$
\ddot{\alpha}^{o}-a_{2} \dot{\alpha}^{o}-a_{1} \alpha^{o}=b_{2} \sigma \xi(t),
$$

where $a_{1}=-\left(\delta^{2}+\theta^{2}\right), a_{2}=-2 \delta, b_{2}=\sqrt{2\left(\delta^{2}+\theta^{2}\right)}$, and $\xi(t)$ is the Gaussian white noise, simulated by the expression

$$
\xi(t)=\sqrt{\frac{2 \delta}{\Delta}} \epsilon_{\Delta}(t) .
$$

Here $\epsilon_{\Delta}(t)=\epsilon_{i}$, and $t \in[i \Delta,(i+1) \Delta]$, where $\epsilon_{i}$ is a sequence of normally distributed uncorrelated numbers with zero mean value and $\left\langle\epsilon^{2}\right\rangle=1 ; \Delta=\Delta t$ is the time step.

Let us consider some results for the viscoelastic plate, obtained at $\varepsilon=0.1, L=0.5, \chi=0.1, \delta=0.5$, $\theta=\omega=1.4$, and $\Delta t=0.1$ by means of the numerical solution of (10) by the fourth-order Runge-Kutta 
method. The number of increments $n$ and initial conditions in all cases were assumed to be the same, namely, $n=10^{4}$ and $z_{1}(0)=1.0, z_{2}(0)=z_{3}(0)=z_{4}(0)=0$.

Figure 2 shows the most typical trajectories of the plate motion on the phase plane $z_{1} \sim z_{2}$. For each of these trajectories the limits of the variation of values $z_{1}$ and $z_{2}$ are indicated. These results can be explained as follows.

If the load is deterministic and constant with time, then the quantity $z$, corresponding to an equilibrium state of the plate, can be found from the cubic equation

$$
\left(1-\alpha_{0}\right) z+0.34125 z^{3}=0 .
$$

If $\alpha_{0}<1$, then this equation has only one root, $z=0$, which corresponds to the unbent equilibrium configuration of the plate, and this state is known to be stable. If $\alpha_{0}>1$, then (13) has three roots,

$$
z_{(1)}=0, \quad z_{(2),(3)}= \pm \sqrt{\frac{\alpha_{0}-1}{0.34125}} .
$$

It can be shown that the solution $z_{(1)}$ is unstable and nontrivial while solutions $z_{(2)}$ and $z_{(3)}$ are stable. The quantity $\alpha_{0}=1$ is critical for an elastic plate.

a)

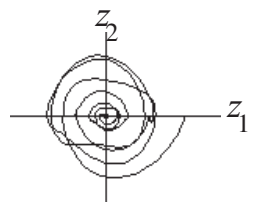

$-0.513 \leqq z_{1} \leqq 1.000$

$-0.520 \leqq z_{2} \leqq 0.507$ b)

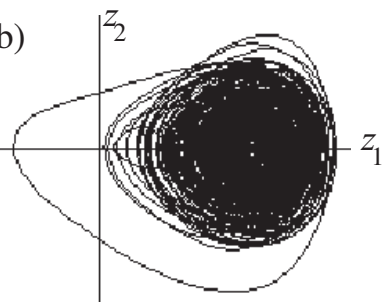

$-0.876 \leqq z_{1} \leqq 2.423$

$-1.834 \leqq z_{2} \leqq 1.458$

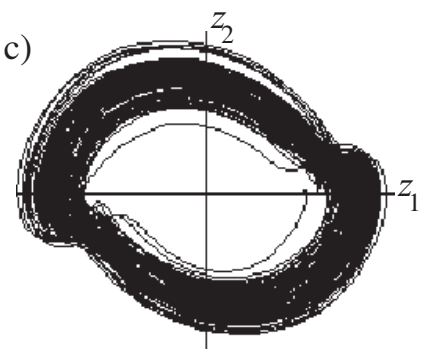

$-1.927 \leqq z_{1} \leqq 1.841$

$-1.691 \leqq z_{2} \leqq 1.948$
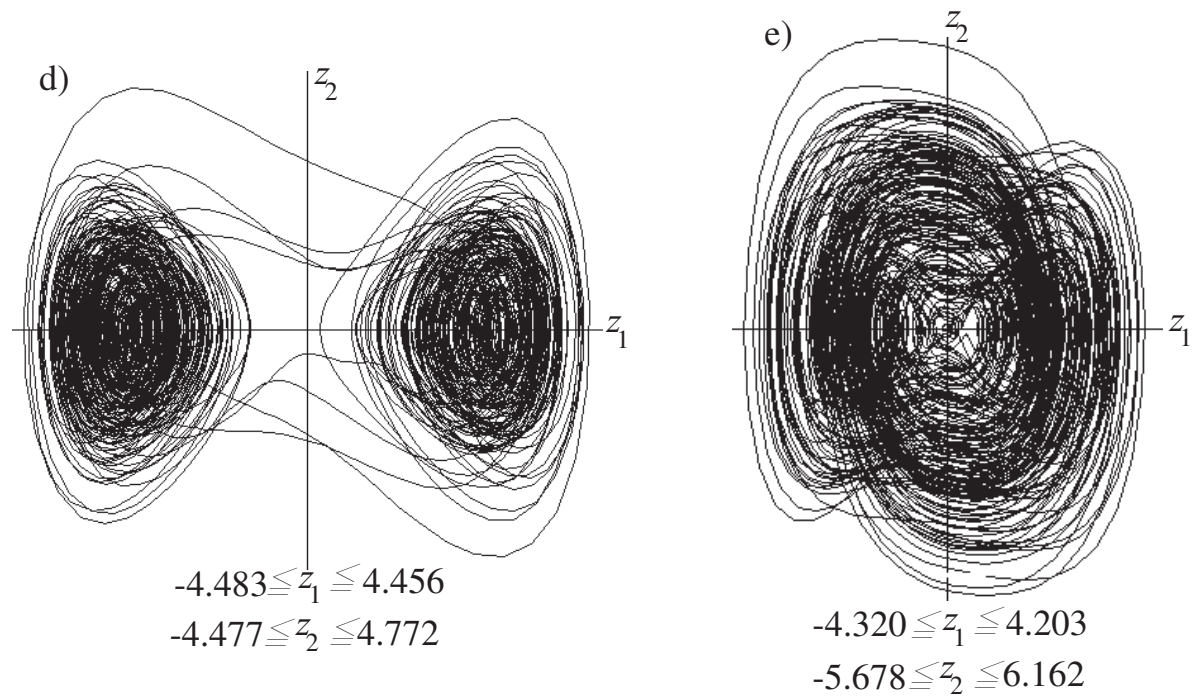

Figure 2. Trajectories of the viscoelastic plate motion in the phase plane. 


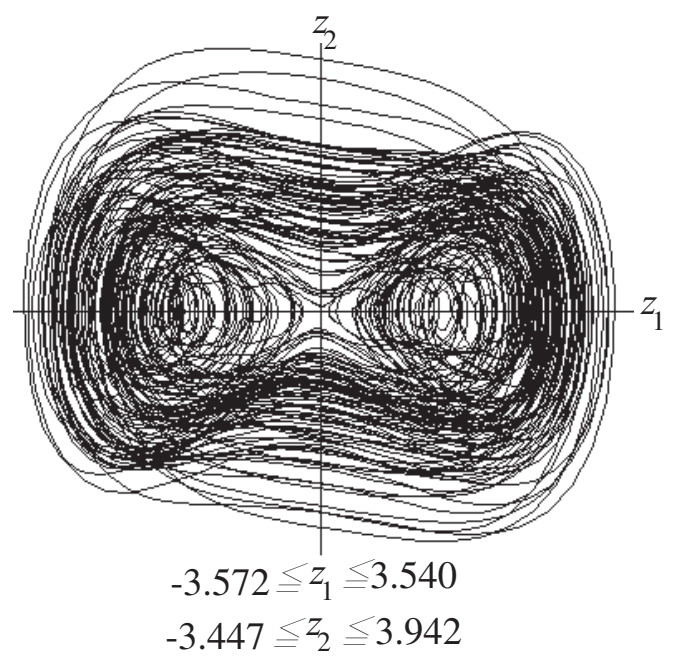

Figure 3. The trajectory of the elastic plate motion in the phase plane.

The situation is similar in a viscoelastic plate, but in this case the critical value of the parameter $\alpha_{0}$ is equal to $1-\bar{R}$, where

$$
\bar{R}=\int_{0}^{\infty} R(\theta) d \theta .
$$

The results for the dynamic (deterministic or stochastic) statement of the problem are similar. Indeed, if parameters $\alpha_{0}, \alpha_{1}$, and $\sigma$ are sufficiently small, then, obviously, oscillations are performed in the neighborhood of the trivial equilibrium state. This statement is confirmed by the plots in Figure $2 \mathrm{a}$, obtained at $\alpha_{0}=0, \alpha_{1}=1.0$, and $\sigma^{2}=0.09$, and dampened with time.

But if the same parameters $\alpha_{0}, \alpha_{1}$, and $\sigma$ are sufficiently large, then the plate motion becomes much more diverse. The plate can oscillate in the neighborhood of a certain equilibrium (see Figure $2 b$ at $\alpha_{0}=1.0, \alpha_{1}=0$, and $\sigma^{2}=0.04$ ) or jump between two equilibria (see Figure $2 \mathrm{c}$ at $\alpha_{0}=0.5, \alpha_{1}=1.0$, and $\sigma^{2}=0.01$; and Figure $2 \mathrm{~d}$ at $\alpha_{0}=0.5, \alpha_{1}=0.5$, and $\sigma^{2}=0.25$ ); moreover, in some cases the plate motion is chaotic (see Figure $2 \mathrm{e}$ at $\alpha_{0}=1.0, \alpha_{1}=1.0$, and $\sigma^{2}=0.25$ ). It should be said that in such cases with increasing time the solution of the nonlinear equations becomes steady-state and different initial conditions may lead to different steady-state solutions.

The effect of the viscous properties of the material on the behavior of the plate can be estimated with help of the trajectory, shown in Figure 3, obtained with the same input data and the same realization of the process $\alpha^{o}(t)$, as in Figure 2d, and of the charts of variation deflections $z_{1}$ with time (see Figure 4), which correspond to the same elastic and viscoelastic plates. In Figure 5 the histograms of the value $z_{1}$ are shown corresponding to the same input data and obtained at $n=10^{5}\left(\left\langle z_{1}\right\rangle\right.$ and $\sigma_{1}$ are the mean value and the mean square scattering of $z_{1}$, respectively). As seen the viscous properties of the material lead to the noticeable modification of the trajectories on the phase plane and with time. A more detailed analysis of these trajectories shows that the motion of the elastic plate is chaotic (unstable) while that of the viscoelastic plate is asymptotically stable. These histograms have the same form for different times if they are far enough from initial time $t=0$; this fact verifies the stationary state of the plate motion. 
a)

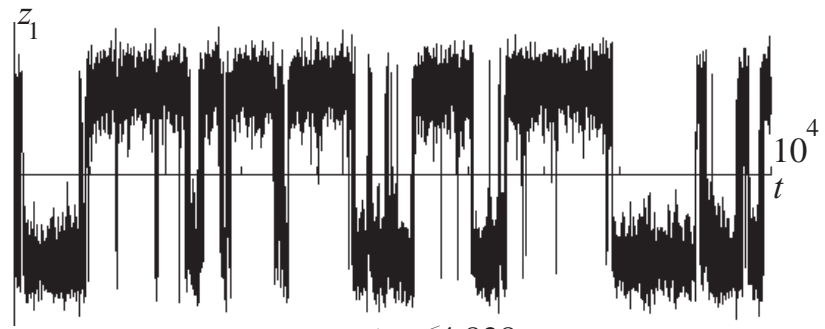

$-4.734 \leqq z_{1} \leqq 4.838$

b) $z_{1}$

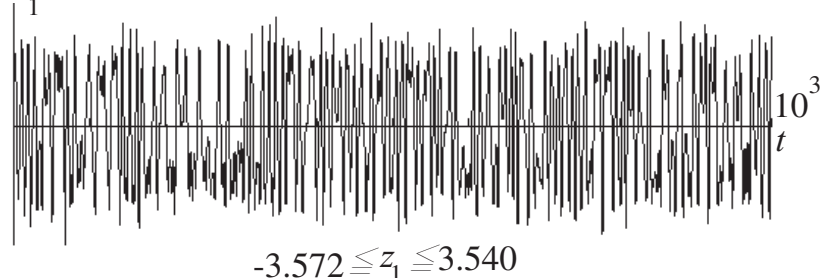

Figure 4. Realizations of the generalized displacement $z_{1}=f(t)$ in the (a) viscoelastic and (b) elastic plates.
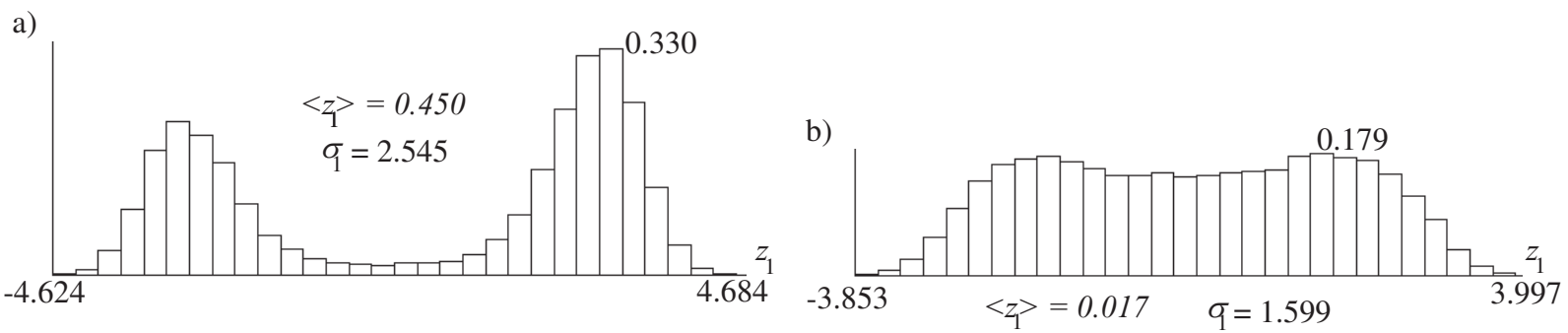

Figure 5. Histograms of the value $z_{1}$ for the (a) viscoelastic and (b) elastic plates.

\section{Stability of the unperturbed motion of the system}

For the analysis of the stability of the unperturbed motion of the system, described by Equation (9), let us consider the perturbed motion, caused by perturbations of the initial conditions.

In the case of perturbed motion the solution of (1) has the form $y=z+\delta z$, that is,

$$
\dot{\boldsymbol{y}}=\boldsymbol{F}(\boldsymbol{y}, \alpha(t), t)
$$

where $z=\left\{z_{1}, z_{2}, \ldots, z_{n}\right\}^{T}$ is the vector of unknowns, corresponding to the unperturbed motion of the system; the vector $\boldsymbol{y}=\left\{y_{1}, y_{2}, \ldots, y_{n}\right\}^{T}$, corresponding to the perturbed motion; and the vector of perturbations $\delta z=\left\{\delta z_{1}, \delta z_{2}, \ldots, \delta z_{n}\right\}^{T}$. Let us expand the right-hand side of (14) by the Taylor series in the neighborhood of the solution $z(t)$ :

$$
\boldsymbol{F}(z+\delta z, \alpha(t), t)=\boldsymbol{F}(z, \alpha(t), t)+\boldsymbol{F}^{\prime}(z, \alpha(t), t) \delta z+\cdots
$$


Restricting this expansion to the two first terms and taking into account (1), we obtain a linearized equation:

$$
\delta \dot{z}=F^{\prime}(z, \alpha(t), t) \delta z
$$

The solution of the equation should satisfy the initial condition $\delta z_{0}(0)=\delta z_{0}, \delta z_{0}=\left\{\delta z_{01}, \delta z_{02}, \ldots, \delta z_{0 n}\right\}^{T}$.

For the estimation of the stability of the system the method of top Lyapunov exponents is used, which is calculated for each pair consisting of a realization $\alpha(t)$ and the corresponding realization of the process $z(t)$. Further, the stability is treated as stability with respect to the statistical moments.

- The solution $\delta z(t) \equiv 0$ is called $p$-stable if, for any $\varepsilon>0$, a $\Delta>0$ can be found such that at $t \geq 0$ and $\left|\delta z_{i}(0)\right|<\Delta(i=1,2, \ldots, n)$, where $n$ is the number of first-order differential equations in (15) and $\left|\left\langle\delta z_{i}^{p}(t)\right\rangle\right|<\varepsilon$.

- The solution $\delta z(t) \equiv 0$ is called asymptotically $p$-stable if it is $p$-stable and, in addition, for a small enough $\left|\delta z_{i}(0)\right|(i=1,2, \ldots, n)$,

$$
\lim _{t \rightarrow \infty}\left|\left\langle\delta z_{i}^{p}(t)\right\rangle\right|=0
$$

At $p=1$ stability takes place in the mean (with respect to the mathematical expectation), and at $p=2$ there is stability in the mean square.

The growth of the vector $\delta z(t)$ can be estimated with the help of the top Lyapunov exponent $\lambda$, which is defined by the expression

$$
\lambda=\lim _{t \rightarrow \infty} \frac{1}{t} \ln \frac{\|\delta z(t)\|}{\|\boldsymbol{\delta} z(0)\|},
$$

where $\|\delta z(t)\|,\|\delta z(0)\|$ is the norm of the vector $\delta z(t)$ in a Euclidean space at time $t$ and at initial time $t=0$.

The value $\lambda$ can be found numerically with help of the method proposed in [Benettin et al. 1980a; Benettin et al. 1980b]. With this purpose in mind let us divide a large enough time interval [0, $t]$ into $m$ equal intervals, so $\Delta t=t_{j+1}-t_{j}(j=1,2, \ldots, m)$.

Let us assume that (15) is deterministic and that at $t=t_{j}$ the norm of the vector $\left\|\boldsymbol{\delta} z\left(t_{j}\right)\right\|$ is of unit length. Using this vector as the vector of initial conditions, let us obtain the solution of the system (15) for time $t_{j+1}$ with the norm $\| \boldsymbol{\delta} z\left(t_{j+1} \|=d_{j+1}\right.$. Continuing the solution of the system (15) with new initial conditions $\delta z_{i 0}\left(t_{j+1}\right) / d_{j+1}$, we will find the sequence of values $d_{j}$, and then the top Lyapunov exponent can be found as the limit

$$
\lambda=\lim _{m \rightarrow \infty} \frac{1}{m \Delta t} \sum_{i=1}^{m} \ln d_{j} .
$$

Because the system of equations for the statistical moments of functions $\delta z_{i}(t)$ in the case of colored noises $\alpha^{o}(t)$ cannot be obtained in a closed form, let us find it using the method of statistical simulation [Potapov 1997; 1999].

The estimation of statistical moments $\left\langle\delta z_{i}^{p}\right\rangle$ for times $t_{j}$ can be obtained as the result of a statistical averaging of the values $\left\langle\delta z_{i}^{p}\right\rangle$, found from (15) for a sufficiently large number of realizations $q$ :

$$
\left\langle\tilde{\delta} z_{i}^{p}\left(t_{j}\right)\right\rangle=\frac{1}{q} \sum_{m=1}^{q}\left[\delta z_{i}^{p}\left(t_{j}\right)\right]^{(m)},
$$


where $\left[\delta z_{i}^{p}\left(t_{j}\right)\right]^{(m)}$ is the quantity $\delta z_{i}^{p}\left(t_{j}\right)$ corresponding to $m$-th realization of the solution of (15).

Assume that the norm of the vector

$$
\left\langle\delta z^{p}\left(t_{k}\right)\right\rangle=\left\{\left\langle\delta z_{1}^{p}\left(t_{k}\right)\right\rangle,\left\langle\delta z_{2}^{p}\left(t_{k}\right)\right\rangle, \ldots,\left\langle\delta z_{n}^{p}\left(t_{k}\right)\right\rangle\right\}^{T}
$$

in the Euclidean space for time $t_{k}$ is of unit length. The norm of the vector $\left\langle\delta z(t)^{p}\right\rangle$ becomes equal to $\tilde{d}_{k+1}$ at time $t_{k+1}=t_{k}+\Delta$. Furthermore, the system of equations (15) is solved for each realization of the matrix $\boldsymbol{F}^{\prime}(z, \alpha(t), t)$ with initial conditions

$$
\delta z_{i 0}\left(t_{k+1}\right)=\frac{\delta z_{i}\left(t_{k+1}\right)}{\left(\tilde{d}_{k+1}\right)^{1 / p}} .
$$

Repeating the stated procedure we obtain the sequence of values $\tilde{d}_{k}$, with the help of which the estimation of the top Lyapunov exponent can be found:

$$
\tilde{\Lambda}=\lim _{l \rightarrow \infty} \frac{1}{l \Delta} \sum_{k=1}^{l} \ln \tilde{d}_{k} .
$$

Let us illustrate the investigation of the stability of geometric nonlinear systems by the example of the above plate.

Equation (9), linearized with respect to perturbations $\delta z$, has the following form:

$$
\delta z^{\prime \prime}+2 \varepsilon \delta z^{\prime}+\left[(1-\alpha-\boldsymbol{R})+0.34125\left(3 z^{2}-2 z \boldsymbol{R} z\right)\right] \delta z-\delta z \boldsymbol{R} z^{2}=0 .
$$

We introduce the new variables

$$
z_{5}=\delta z, \quad z_{6}=\delta z^{\prime}, \quad z_{7}=\int_{0}^{t} \chi L e^{-\chi(t-\tau)} \delta z(\tau) d \tau, \quad z_{8}=\int_{0}^{t} \chi L e^{-\chi(t-\tau)} z(\tau) \delta z(\tau) d \tau ;
$$

hence we can write (18) as a system of first-order differential equations

$$
\begin{array}{ll}
z_{5}^{\prime}=z_{6}, & z_{6}^{\prime}=-2 \varepsilon z_{6}-(1-\alpha) z_{5}+z_{7}-0.34125\left(3 z_{1}^{2} z_{5}-2 z_{1} z_{8}+z_{4} z_{5}\right), \\
z_{7}^{\prime}=\chi L z_{5}-\chi z_{7}, & z_{8}^{\prime}=\chi L z_{1} z_{5}-\chi z_{8} .
\end{array}
$$

If the plate is subjected to a deterministic periodic load, then at some values of the input parameters the motion of the plate can be chaotic [Guckenheimer and Holmes 1996].

This is confirmed by the trajectory of the motion of the elastic plate on the phase plane $z_{1} \sim z_{2}$, shown in Figure 6a, which is obtained at $\varepsilon=0,1 ; \alpha_{0}=0.5 ; \alpha_{1}=2.0 ; \omega=1,4 ; \Delta t=0.1$; and number of increments $n=10^{4}$. The value of the top Lyapunov exponent at $t=6.10^{4}$ is $\lambda=0.165$ (see Figure 7a), which confirms the instability of the plate.

If we impose on the deterministic load a random noise in the form of a Gaussian stationary process $\alpha^{o}(t)$ with characteristics $\sigma^{2}=0.01, \delta=0.5$, and $\theta=1.4$, then the plate becomes stable with respect to statistical moments of the first, $\lambda_{1}=-0.082$ (see Figure 7b), and second, $\lambda_{2}=-0.164$ (see Figure $7 \mathrm{c}$ ), orders. These estimates of the top Lyapunov exponents are obtained as a result of averaging the results of 20 realizations at $t=6.10^{4}$. One of realizations of the trajectories on the phase plane in this case, obtained at $n=10^{4}$, is shown in Figure $6 \mathrm{~b}$. 

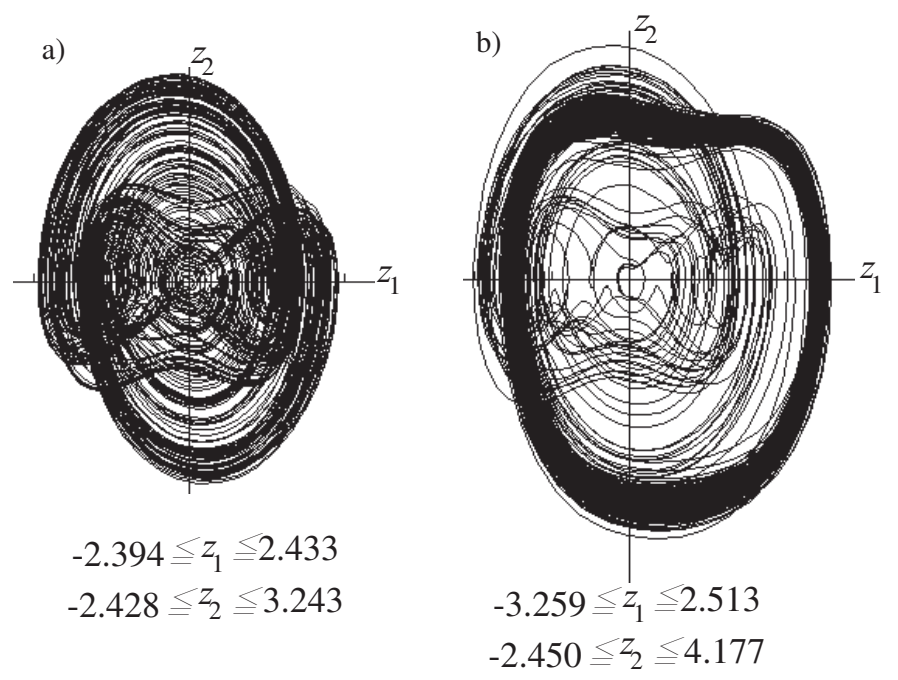

Figure 6. Trajectories of the elastic plate motion in the phase plane under (a) deterministic and (b) stochastic treatments of the problem.

a)

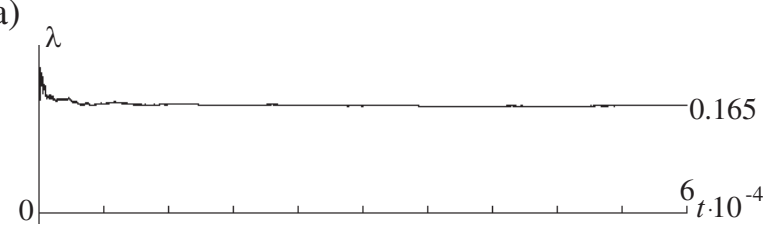

c) $\lambda$

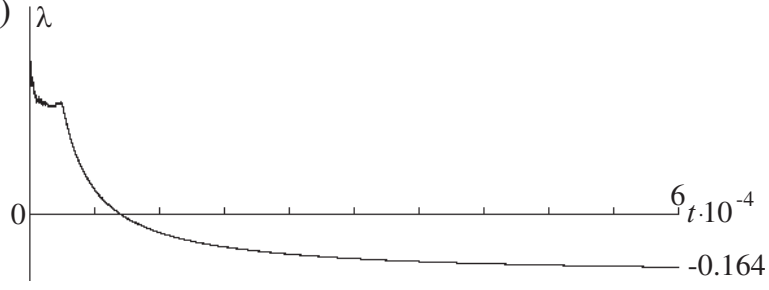

b) $1 \lambda$

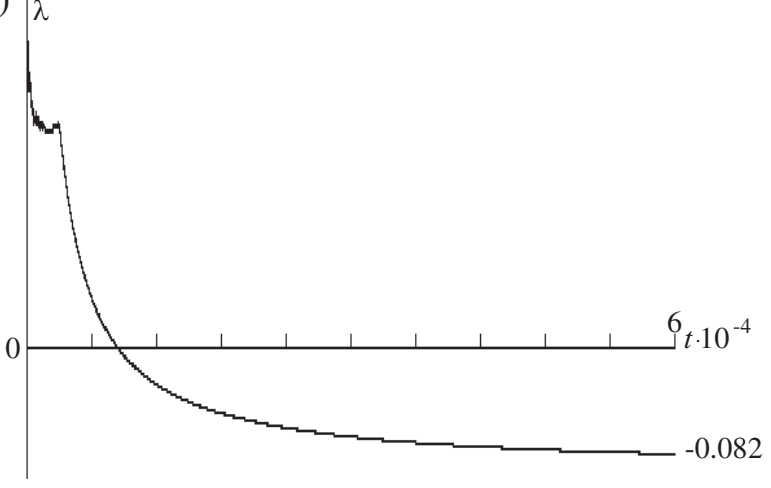

Figure 7. Changes in the estimate of the top Lyapunov exponent for the elastic plate under (a) deterministic and (b and c) stochastic treatments of the problem.

If we increase the time interval to $10^{5}$, the same values result in quantities $\lambda_{1}=-0.088$ and $\lambda_{2}=$ -0.180 . At finally, if the number of realizations is assumed to be equal to 40 , then at $t=10^{5}$ we will find $\lambda_{1}=-0.087$ and $\lambda_{2}=-0.177$.

If the material of the plate has a small viscosity, then the indicated effect remains. This is confirmed by the data, shown in the tables at the top of the next page. The results shown in Table 1 are obtained for the plate under the action of a deterministic load, with and without random noise (with characteristics $\sigma^{2}=0.01, \delta=0.5$, and $\theta=1.4$ ). These quantities of the top Lyapunov exponents are found as a result of data processing of 40 realizations at $t=10^{5}$.

The presented results show that the calculation of top Lyapunov exponent estimates demands a lot of steps with respect to time. In such situations questions about the exactness of the numerical solution 


\begin{tabular}{|llr|}
\hline$L$ & $\chi$ & $\lambda$ \\
0 & 0 & 0.166 \\
0.1 & 0.1 & 0.156 \\
0.5 & 0.1 & 0.131 \\
0.1 & 0.5 & 0.151 \\
0.5 & 0.5 & -0.149 \\
\hline
\end{tabular}

\begin{tabular}{|ll|rr|rr|}
\hline$L$ & $\chi$ & $\lambda_{1}^{(4)}$ & $\lambda_{2}^{(4)}$ & \multicolumn{1}{c|}{$\lambda_{1}^{(5)}$} & \multicolumn{1}{c|}{$\lambda_{2}^{(5)}$} \\
0 & 0 & -0.087 & -0.177 & -0.085 & -0.179 \\
0.1 & 0.1 & -0.086 & -0.172 & -0.086 & -0.167 \\
0.5 & 0.1 & 0.132 & 0.260 & 0.132 & 0.259 \\
0.1 & 0.5 & 0.140 & 0.273 & 0.136 & 0.284 \\
0.5 & 0.5 & -0.146 & -0.291 & -0.146 & -0.291 \\
\hline
\end{tabular}

Table 1. Results obtained for the plate under the action of a deterministic load (left) and under a deterministic load and random noise with $\sigma^{2}=0.01, \delta=0.5$, and $\theta=1.4$.

arise. In order to verify the results obtained with the help of the fourth-order Runge-Kutta method, the $\lambda_{1}^{(4)}$ results are shown in Table 1; these are found with the help of the fifth-order Runge-Kutta-Fehlberg results, $\lambda_{1}^{(5)}$ [Fehlberg 1969; Butcher 2003]. Because the algorithm of this method is not widely known we will give a scheme for the numerical solution of the ordinary differential equation $d z / d t=f(t, z)$ :

$$
z_{n+1}=z_{n}+h \sum_{i=1}^{s} b_{i} k_{i}, \quad k_{i}=f\left(t_{n}+c_{i} h, x_{n}+h \sum_{j=1}^{s} a_{i j} k_{j}\right) .
$$

This method is defined by the Butcher tableau, which shows the coefficients of the method as follows:

$$
\begin{array}{c|cccc}
c_{1} & a_{11} & a_{12} & \ldots & a_{1 s} \\
c_{2} & a_{21} & a_{22} & \ldots & a_{2 s} \\
\vdots & \vdots & \vdots & \ddots & \vdots \\
c_{s} & a_{s 1} & a_{s 2} & \ldots & a_{s s} \\
\hline & b_{1} & b_{2} & \ldots & b_{s}
\end{array}
$$

\begin{tabular}{|c|c|c|c|c|c|}
\hline \multicolumn{6}{|l|}{0} \\
\hline $1 / 4$ & $1 / 4$ & & & & \\
\hline $3 / 8$ & $3 / 32$ & $9 / 32$ & & & \\
\hline $12 / 13$ & $1932 / 2197$ & $-7200 / 2197$ & $7296 / 2197$ & & \\
\hline 1 & $439 / 216$ & -8 & $3680 / 513$ & $-845 / 4104$ & \\
\hline $1 / 2$ & $-8 / 27$ & 2 & $-3544 / 2565$ & $1859 / 4104$ & $-11 / 40$ \\
\hline & $16 / 135$ & 0 & $6656 / 12825$ & $28561 / 56430$ & $-9 / 50 \quad 2 / 55$ \\
\hline
\end{tabular}

These coefficients for the Runge-Kutta-Fehlberg version are equal to

The comparison of the corresponding results shows that the fourth-order Runge-Kutta method gives sufficiently correct results.

The stabilizing effect of the unstable system was discovered for the first time in the investigation of the stability of the first-order differential equation [Leibowitz 1963; Hasminskir 1980]

$$
\dot{X}=(b+\sigma \xi) X,
$$

where $b$ and $\sigma$ are constants, and $\xi$ is a Gaussian white noise, introduced by Ito. 
The trivial solution of Equation (20) is stable at $b<\sigma^{2} / 2$, which has been considered as physically contradictive [Hasminskiı̌ 1980]. On this basis the conclusion was made that the white noise in Ito's sense is "physically unrealizable".

If $\xi(t)$ is a white noise in Stratonovich's sense, then the effect of the stabilization disappears [Hasminskiı 1980], and it has been remarked that the unstable deterministic system $\dot{x}=b x(b>0-$ const.) cannot be stabilized by a physically realizable perturbation of its parameter.

However, Hasminskiı̌ [1980] has shown that for higher-order systems of differential equations Stratonovich white noise can stabilize an unstable deterministic system. In particular, the trivial solution of the second-order equation $\ddot{z}+(k+\sigma \chi) \dot{z}+\omega^{2} z=0$, where $k$ and $\omega^{2}$ are constants, at definite quantities of the parameter $\sigma$ can be stable, although the same solution of the deterministic equation (at the same magnitudes of values $k$ and $\left.\omega^{2}\right) \ddot{z}+k \dot{z}+\omega^{2} z=0$ is unstable.

It should be said that white noises in both Ito's and Stratonovich's senses are mathematical idealizations, which cannot be realized physically (as physical processes with unlimited power doesn't exist). From this point of view the smoothing in the mean square process, used in the present paper, can be considered a physically realizable process.

Arnold et al. [1983] showed that deterministic differential equations can be stabilized (in sense of the almost sure stability) by stochastic wide-band stationary processes. The result, obtained for the plate in the present paper, demonstrates that a physically realizable (colored) noise can render a stabilizing effect (in sense of stability with respect to statistical moments) in the analysis of the stability of elastic and viscoelastic systems, the motion of which is described by nonlinear differential equations.

\section{Conclusion}

In the present paper an effective method of investigation of nonlinear oscillations and the stability of elastic and viscoelastic systems at stochastic excitations is proposed. Loads are assumed to be in the form of Gaussian random stationary processes with rational spectral densities (colored noises). The method is based on the numerical simulation of random processes, the numerical solution of differential equations, describing the motion of the considered system, and on the calculation of the top Lyapunov exponent. It is shown that the viscous properties of the material have an essential effect on its nonlinear oscillations. It is remarked that in some cases the addition of a stochastic noise on the deterministic periodic excitation can have a stabilizing effect on the motion of elastic and viscoelastic plates.

\section{References}

[Arnold et al. 1983] L. Arnold, H. Crauel, and V. Wihstutz, "Stabilization of linear systems by noise", SIAM J. Control Optim. 21:3 (1983), 451-461.

[Benettin et al. 1980a] G. Benettin, L. Galgani, A. Giorgilli, and J.-M. Strelcyn, "Liapunov characteristic exponents for smooth dynamical systems and for Hamiltonian systems; a method for computing all of them. 1: Theory", Meccanica (Milano) 15:1 (1980), 9-20.

[Benettin et al. 1980b] G. Benettin, L. Galgani, A. Giorgilli, and J.-M. Strelcyn, "Liapunov characteristic exponents for smooth dynamical systems and for Hamiltonian systems; a method for computing all of them. 2: Numerical application", Meccanica (Milano) 15:1 (1980), 21-30.

[Butcher 2003] J. C. Butcher, Numerical methods for ordinary differential equations, Wiley, Chichester, 2003. 
[Dimentberg 1980] M. F. Dimentberg, Нелинеиные стохастические задачи механических колебании, Nauka, Moscow, 1980.

[Dimentberg 1988] M. F. Dimentberg, Statistical dynamics of nonlinear and time-varying systems, Mechanical Engineering Research Studies. Engineering Dynamics Series 5, Research Studies Press, Taunton, 1988.

[Fehlberg 1969] E. Fehlberg, "Klassische Runge-Kutta-Formeln fünfter und siebenter Ordnung mit Schrittweiten-Kontrolle", Computing (Arch. Elektron. Rechnen) 4:2 (1969), 93-106.

[Guckenheimer and Holmes 1996] J. Guckenheimer and P. Holmes, Nonlinear oscillations, dynamical systems, and bifurcations of vector fields, Springer, New York, 1996.

[Hasminskiı̌ 1980] R. Z. Hasminskiü, Stochastic stability of differential equations, Monographs and Textbooks on Mechanics of Solids and Fluids: Mechanics and Analysis 7, Sijthoff \& Noordhoff, Alphen aan den Rijn, 1980.

[Leibowitz 1963] M. A. Leibowitz, "Statistical behavior of linear systems with randomly varying parameters", J. Math. Phys. 4:6 (1963), 852-858.

[Potapov 1997] V. D. Potapov, "Numerical method for investigation of stability of stochastic integro-differential equations", Appl. Numer. Math. 24:2-3 (1997), 191-201.

[Potapov 1999] V. D. Potapov, Stability of stochastic elastic and viscoelastic systems, Wiley, Chichester, 1999.

[Shalygin and Palagin 1986] A. C. Shalygin and Y. I. Palagin, Applied methods of statistical simulation, Mashinostroenie, Leningrad, 1986.

[Simiu 2002] E. Simiu, Chaotic transitions in deterministic and stochastic dynamical systems: applications of Melnikov processes in engineering, physics, and neuroscience, Princeton University Press, Princeton, NJ, 2002.

[Vol'mir 1967] A. S. Vol'mir, Устоичивость деформируемых систем, Nauka, Moscow, 1967. In Russian; translated as Stability of deformable systems, Ft. Belvoir Defense Technical Information Center, 1970.

Received 3 Dec 2008. Revised 22 Jun 2009. Accepted 20 Aug 2009.

VADIM D. POTAPOV: potapov@icnmic.msk.ru

Department of Structural Mechanics, Moscow State University of Means Communication, Obraztsov street, 15,

Moscow 127994, Russia 


\title{
JOURNAL OF MECHANICS OF MATERIALS AND STRUCTURES
}

\author{
http://www.jomms.org
}

\author{
Founded by Charles R. Steele and Marie-Louise Steele

\section{EDITORS} \\ Charles R. STEele \\ DAVIDE BIGONI \\ IWONA JASIUK \\ YASUHIDE SHINDO \\ Stanford University, U.S.A. \\ University of Trento, Italy \\ University of Illinois at Urbana-Champaign, U.S.A. \\ Tohoku University, Japan
}

\section{EDITORIAL BOARD}

H. D. BUI École Polytechnique, France

J. P. CARTER University of Sydney, Australia

R. M. Christensen Stanford University, U.S.A.

G. M. L. GLADWELL University of Waterloo, Canada

D. H. HodGES Georgia Institute of Technology, U.S.A.

J. HUTCHINSON Harvard University, U.S.A.

C. HwU National Cheng Kung University, R.O. China

B. L. KariHaloo University of Wales, U.K.

Y. Y. KIM Seoul National University, Republic of Korea

Z. Mroz Academy of Science, Poland

D. PAMPlonA Universidade Católica do Rio de Janeiro, Brazil

M. B. RUBIN Technion, Haifa, Israel

A. N. SHUPIKov Ukrainian Academy of Sciences, Ukraine

T. TARNAI University Budapest, Hungary

F. Y. M. WAN University of California, Irvine, U.S.A.

P. WRIGGERS Universität Hannover, Germany

W. YANG Tsinghua University, P.R. China

F. ZIEGLER Technische Universität Wien, Austria

\section{PRODUCTION}

\section{Paulo Ney de Souza Production Manager \\ SheIla Newbery Senior Production Editor \\ SILVIO LEVY Scientific Editor}

See inside back cover or http://www.jomms.org for submission guidelines.

JoMMS (ISSN 1559-3959) is published in 10 issues a year. The subscription price for 2010 is US $\$ 500 /$ year for the electronic version, and \$660/year (+\$60 shipping outside the US) for print and electronic. Subscriptions, requests for back issues, and changes of address should be sent to Mathematical Sciences Publishers, Department of Mathematics, University of California, Berkeley, CA 94720-3840.

JoMMS peer-review and production is managed by EditFLOW ${ }^{\mathrm{TM}}$ from Mathematical Sciences Publishers.

PUBLISHED BY

mathematical sciences publishers

http://www.mathscipub.org

A NON-PROFIT CORPORATION

Typeset in LATEX

CCopyright 2010. Journal of Mechanics of Materials and Structures. All rights reserved. 


\title{
Journal of Mechanics of Materials and Structures
}

\author{
Volume 5, No. 2 February 2010
}

A critical analysis of interface constitutive models for the simulation of delamination in composites and failure of adhesive bonds

Anton Matzenmiller, Sebastian Gerlach and Mark Fiolka

185

Computational studies of collagen fibril biominerals using a virtual internal bond

model with extrinsic length scale

Ganesh Thiagarajan and Kavita Deshmukh

The simulation of stochastically excited viscoelastic systems and their stability

VADIM D. POTAPOV

Fundamental solutions for an inhomogeneous cross-anisotropic material due to horizontal and vertical plane strain line loads

Cheng-Der Wang, Jia-YAN Hou and WeI-Jer WANG

Mechanical and fracture analysis of welded pearlitic rail steels

Aldinton Allie, Heshmat A. Aglan and Mahmood Fateh

Rate dependence of indentation size effects in filled silicone rubber

Ramanjaneyulu V. S. TATIRAJU and ChUng-SOUK HAN

A novel application of a laser Doppler vibrometer in a hèalth monitoring system

DAVOOD REZAEI and FARID TAHERI

Energy absorption of a helicoidal bistable structure

Seubpong Leelavanichkul, Andrej Cherkaev, Daniel O. Adams

and FLORIAN SOLZBACHER

Decay properties of solutions of a Mindlin-type plate model for rhombic systems

Francesca Passarella, Vincenzo Tibullo and VitTorio Zampoli

A consistent refinement of first-order shear deformation theory for laminated composite and sandwich plates using improved zigzag kinematics Alexander Tessler, Marco Di Sciuva and Marco Gherlone 